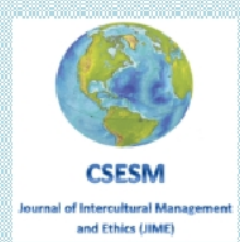

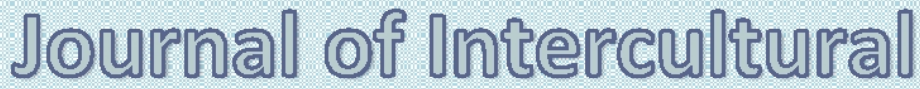

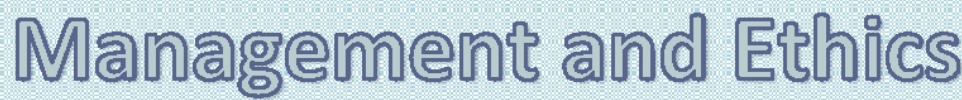

\author{
IOME
}

ISSN 2601 - 5749, ISSN-L 2601 - 5749

\section{published by zy \\ Center for Socio-Economic Studies and Multiculturalism \\ lasi, Romania \\ Waw csesmorg}




\section{Special Editor}

\section{Professor Beatrice Gabriela Ioan, PhD, MD}

Grigore T.Popa University of Medicine and Pharmacy of Iasi, Romania

E-mail: ioanbml@yahoo.com

\section{TABLE OF CONTENT}

Editorial ....

Beatrice Gabriela Ioan

Ethical Approaches on the Mandatory Vaccination in the Pandemic Context (Romania Case)

Andreea-Iulia Someșan, Ion Copoeru

Early Approaches in Management of Sars-Cov-2 Infection 19

Isabela-Ioana Loghin, Adriana-Florina Bahnă, Oana-Manuela Secrieru, Irina-Margareta

Nistor, Irina-Cristina Nicolau, Liviu Jany Prisăcariu, Florin Roșu, Victor Daniel Dorobăţ,

Cristin-Ioan Loghin, Carmen-Mihaela Dorobăț

Giving Birth during the Pandemic. How The Decision to Transform Certain Hospitals In Dedicated Covid-19 Medical Units Impacted Women on Psychological Level

Alexandra Ștefania Nadane

Evangelicalism in Uganda: Implications for Public Health and Bioethics

Sana Loue, Francis Bajunirwe

The Contribution of Ethics to the Development of the Healthcare System

Cornelia Margareta Găşpărel

Iatrogenesis Induced by Risk Reduction in Health Care

Mircea Gelu Buta

Ethical Contributions in Preserving the Dignity of the Terminal Patient

Elena Toader, Andreea Decusara, Mirela Piscuc, Tudor Winsinger

Ethical Aspects of the Institutionalization Process of Children from Outbreaks of

Tuberculosis

Rodica Gramma, Elena Cernăuțeanu, Adriana Paladi

Profession, Vocation, Mission or Work. The Ancient Physician and the Contemporary

Physician-Parallel Lives

Orsolya Horber, K.Zilahi 


\title{
EARLY APPROACHES IN MANAGEMENT OF SARS-CoV-2 INFECTION
}

Isabela-Ioana Loghin ${ }^{1,2}$, Adriana-Florina Bahnă ${ }^{2}$, Oana-Manuela Secrieru ${ }^{2}$, Irina-Margareta Nistor $^{2}$, Irina-Cristina Nicolau ${ }^{2}$, Liviu Jany Prisăcariu ${ }^{2}$, Florin Roșu ${ }^{1,2}$, Victor Daniel Dorobăţ ${ }^{3}$, Cristin-Ioan Loghin ${ }^{4}$, Carmen-Mihaela Dorobăț ${ }^{2}$

1 "Grigore T. Popa" University of Medicine and Pharmacy, Iasi, Romania

Faculty of Medicine, Department of Infectious Diseases

2 "St. Parascheva" Clinical Hospital of Infectious Diseases, Iasi, Romania

${ }^{3}$ Emergency Clinical Hospital, Bucharest, Romania

4"Alexandru Ioan Cuza" University, Faculty of Orthodox Theology, Iasi, Romania

* corresponding author, E-mail: isabelabegezsan@yahoo.com

\begin{abstract}
SARS-CoV-2 infection is an infectious pathology that currently poses an exceptional threat to global public health and safety. Of great necessity was the rapid discovery of the effective vaccine, thus leading to the acceleration of ethical approaches to research, including by conducting controlled clinical trials. The therapeutic protocol has always been updated, since the beginning of the pandemic in March 2020, for the correct therapeutic control.

We conducted a study on cases of SARS-CoV-2 infection hospitalized between 15 March and 30 November 2020 in the "St. Parascheva" Clinical Hospital of Infectious Diseases of Iasi, with various forms of the disease.

More than $30 \%$ of cases were severe and critical forms in people with multiple comorbidities, requiring evaluation, monitoring and treatment in the Intensive Care Unit. The treatment regimens, according to the national protocol periodically revised, included association of antivirals, anti-inflammatory medication and immunomodulatory monoclonal antibodies. The risk of various therapeutic associations has always been assessed in order to maximize the effectiveness of treatment and to promote favourable evolutions.

Management of patients with SARS-CoV-2 infection, which also suffered from other diseases, requires interdisciplinary collaboration, while maintaining ethical standards. Nevertheless, the overall effectiveness of immunisation with available SARS-CoV-2 vaccines will be essential to reducing morbidity and mortality in general population.
\end{abstract}

Key words: SARS-CoV-2, COVID-19 pandemic, management, ethical aspects.

\section{Introduction}

Different regions of the globe were affected by the pandemic due to a new coronavirus since January 2020. As of 11 February 2020, data from the World Health Organization (WHO, 2020) have shown that more than 43000 confirmed cases have been identified in 28 countries/regions, with more than $99 \%$ of cases being detected in China. On 30 January 2020, the WHO declared COVID-19 as the sixth public health emergency of international concern (Lai et al., 2020). On March 11, 2020, the World Health Organization has declared the new coronavirus (COVID-19) outbreak a global pandemic (Cucinotta \& Vanelli, 2020).

Managing the pandemic has been a challenge, while most SARS-CoV-2 infections are not severe, a significant percentage of patients require hospitalization, and many fatalities occur, with increased rates of severe and fatal disease among older individuals $(>65$ years 
old) and those with pre-existing medical conditions like cardiovascular disease, obesity, and type 2 diabetes mellitus. Severe cases can progress to respiratory failure associated with diffuse alveolar damage and acute respiratory distress syndrome (Moderbacher et al., 2020).

Managing all the issues related to the infection with SARS-CoV-2 has been a real challenge. Each hospital began to establish its own protocols based on scientific data and information they could gather about the pandemic in the media, in networks or in the experience of other centers (Yepes-Temiño et al., 2021).

The beginning of the pandemic in Romania included a period of almost 16 weeks, when hospital admission of patients diagnosed with SARS-CoV-2 infection was mandatory by law, which was perceived as an abuse and violation of their rights by many of them. Public authorities had made that decision in an attempt to limit the spread of SARS-CoV-2, while also assuming the risk of creating various ethical conflicts, which indeed appeared later on. Ethical conflict is defined as a problem that arises when one considers that the idea of "good," "right" or "doing the right thing" in relation to other people's welfare or best interest is being compromised (Turale et al., 2020). That problem was solved with the cancellation of mandatory hospitalization by the Decree no. 1137/2020 (Ministry of Health, 2020).

Even before the current pandemic, studies had identified a variety of potential ethically conflictive scenarios in hospitals. These scenarios tend to be related to confidentiality, informed consent and respect for the interests of the patients and their autonomy (Laventhal et al., 2020).

Guidelines have been approved worldwide in order to standardize medical treatment of the SARS-CoV-2 infected patients, but the patient's consent was still required. The therapeutic protocol was periodically updated, from the beginning of the pandemic (March 2020), in order to keep up with medical advancements in the field.

During the pandemic, there were countries, such as Italy, with an overwhelmed health system, lacking hospital beds to accommodate all SARS-CoV-2 infected patients, especially in Intensive Care Units (ICUs). These lead to ethical dilemmas, by having doctors assume an ungrateful role, deciding who will be admitted in hospitals/ICUs and who will not. In these situations, the involvement of an ethics committee is very valuable. The ethics committee applies a reflective perspective on the fundamental ethical principles, in order to elucidate and respectively solve the possible ethical problems in different complex clinical scenarios (Blot et al., 2020).

The rapid discovery of an effective and safe vaccine was of great importance and this was possible by conducting controlled clinical trials ethically approved. In Romania, the vaccination campaign started at the end of December 2020 and by January $15^{\text {th }}, 2021$, almost 160.000 health workers were vaccinated (Romanian Government, Communication Group of National Coordination Committee for COVID-19 Vaccination Activities, 2021).

In clinics, the ethical aspects were pursued, especially by informing the patients about the form of the disease, the necessity or recommendation of the hospitalization and explaining the specific therapy, before asking them to provide the informed consent.

\section{Material and Methods}

We performed a retrospective study including patients diagnosed with SARS-CoV-2 infection and hospitalised between March $1^{\text {st }}$ and November $30^{\text {th }}, 2020$, in the "St. Parascheva" Clinical Hospital of Infectious Diseases of Iasi, using information recorded in their medical files. The aim was to highlight the severity of the disease and the challenges raised by the medical and ethical management of patients. We analysed the patients' characteristics, such as age, gender, medical history, disease severity, presence of complications (COVID-19 pneumonia) and evolution, while also taking into consideration the ethical aspects. 


\section{Results}

There were 4101 (1913 men and 2188 women) patients with SARS CoV-2 infection, admitted in our hospital during the mentioned period, with a signed informed consent provided by the patients in the first day of their admission. From those, 3536 patients were from Iasi and 565 from other counties. We found $49 \%$ of cases with severe forms of disease, with $30 \%$ having a critical status (fig.1).

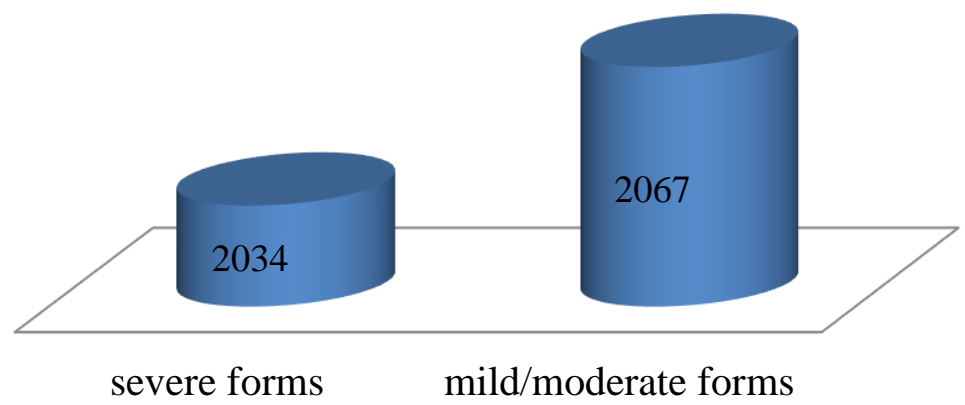

Fig. 1. Severity of SARS CoV-2 infection

The patient profile was: female, aged between 45-54 years old, with medical history of hypertension, obesity or diabetes mellitus (fig. 2, fig. 3, fig. 4).

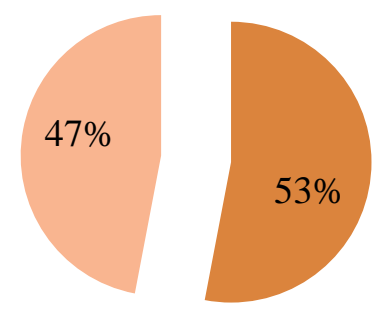

Women

- Men

Fig. 2 Gender distribution of cases with SARS CoV-2 infection

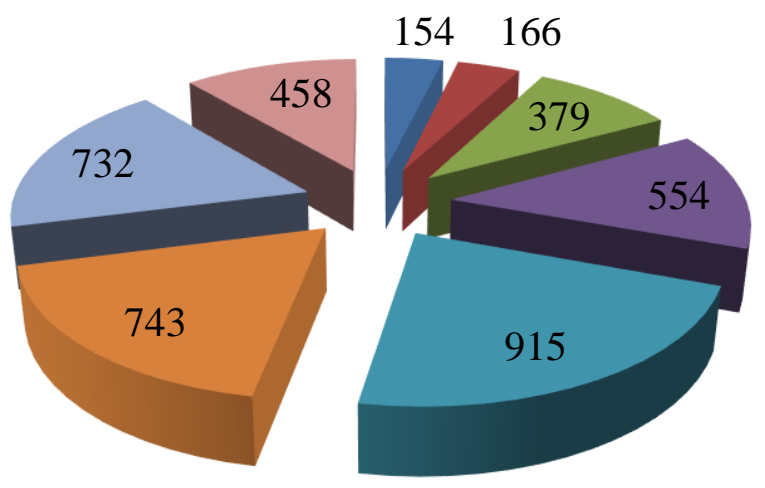

$$
\begin{aligned}
& \square \text { 0-14 years } \\
& \square \text { 15-24 years } \\
& \square \text { 25-34 years } \\
& \square \text { 35-44 years } \\
& \square 5-54 \text { years } \\
& \square \text { 55-64 years } \\
& \square 65-74 \text { years } \\
& \square 75 \text { years }
\end{aligned}
$$

Fig. 3 Age distribution of patients with SARS CoV-2 infection

Out of 4101 patients with SARS-CoV-2 infection, 2438 had other comorbidities (fig.4). 


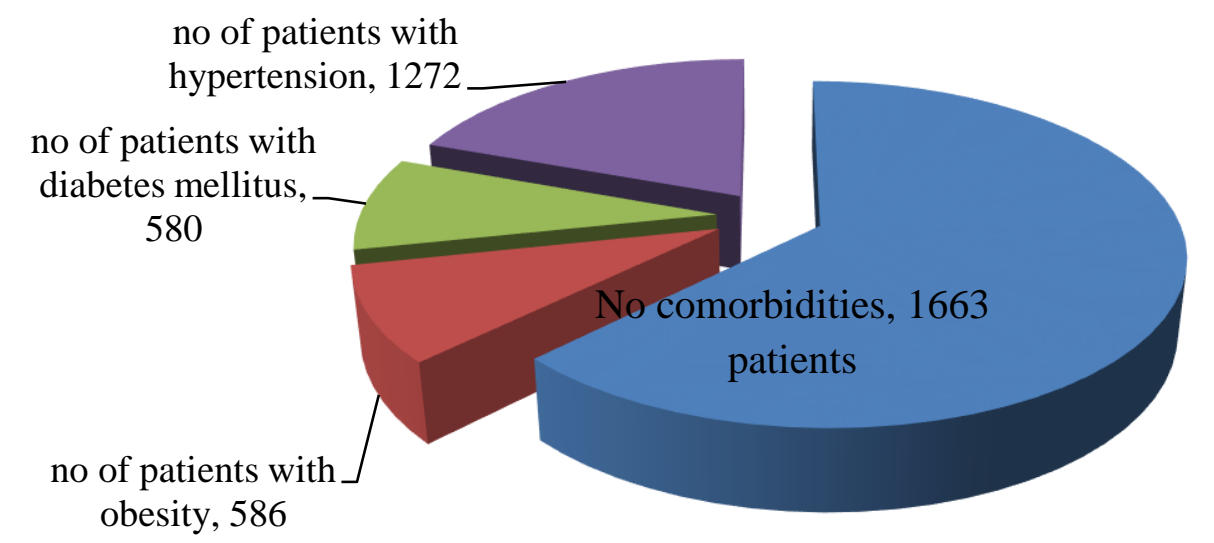

Fig. 4. Patients with SARS-CoV-2 infection and comorbidities

Average length of hospitalisation was 9.62 days. In $46 \%$ of cases, lung damage was associated, developing COVID-19 pneumonia (fig.5).

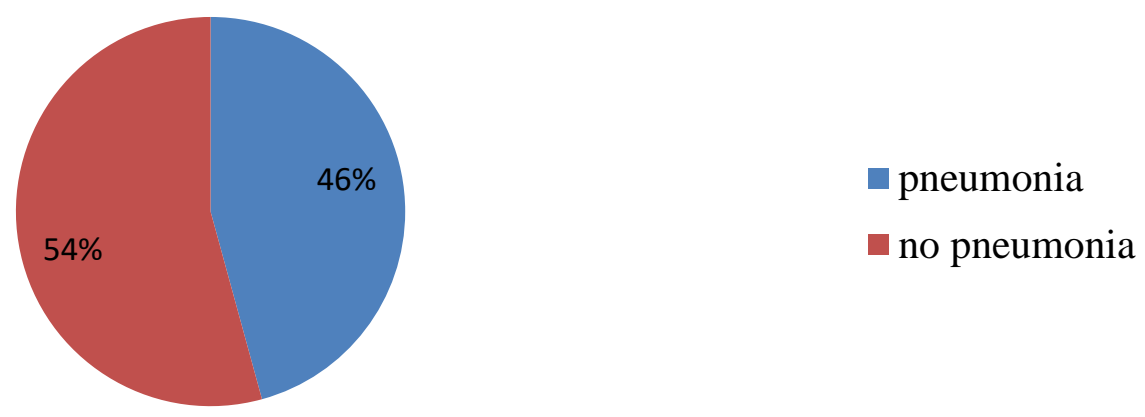

Fig. 5 Patients with SARS-CoV-2 infection and COVID-19 pneumonia

The evolution was unfavourable in $6 \%$ of the cases, leading to the death of 248 patients (146 men, 102 women) (fig.6).

$$
\text { death } \quad \text { favorable evolution }
$$

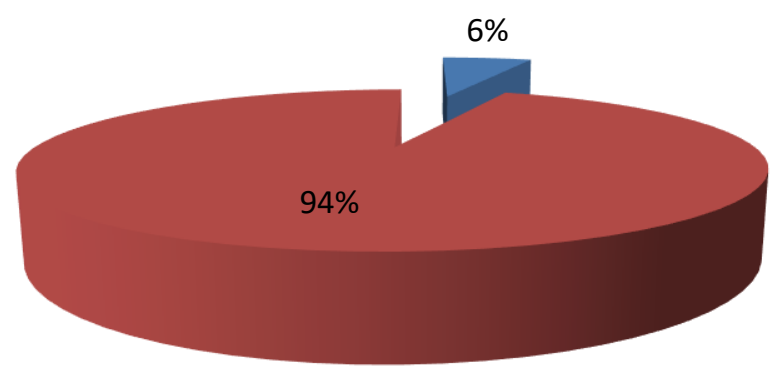

Fig. 6 The evolution of cases with SARS CoV 2 infection

\section{Discussions}

The treatment regimens used in the SARS CoV-2 infected patients, according to the national protocol, included a combination of antivirals, anti-inflammatory medication and immunomodulatory monoclonal antibodies. The patients diagnosed with SARS CoV-2 infection were informed about the necessity of hospitalization and the availability of antiviral 
therapy. The informed consent was signed by the patients before any medical procedure was performed. The ethical aspect was pursued, especially by informing the patients about the form of the disease, the necessity or recommendation of the hospitalization and explaining the specific therapy, before asking them to give their informed consent.

The risk of therapeutic interactions has always been assessed in order to maximize the effectiveness of treatment and the patient's favourable evolution.

Keeping up with medical statistics, which highlighted the magnitude of the pandemic impact, in particular by referring to the number of severe, serious or critical cases, made it easy for medical staff to feel uncertainty regarding the correct course of action, therefore, well-established and updated protocols have been implemented. In our study, almost $50 \%$ of cases had a severe evolution, with $30 \%$ having a critical status. However, the mortality rate was low, only $6 \%$ of cases leading to death.

In the 248 cases of death caused by SARS-CoV-2 infection, we followed the decisions of the National Emergency Department, which established strict rules regarding the handling of the deceased. Therefore, the funeral service was modified, shortened, with an extremely small audience (only a few family members), while also respecting the social distancing recommended by the Decree no. 240/2020 (President of Romania, 2020).

Lack of hospital beds made it necessary to prioritize the cases: admitting those most likely to survive the current illness, or to live the longest after recovery (considering the comorbidities) (Robert et al., 2020). All these things could have been perceived as a lottery, but doctors' decisions were guided by prioritization strategies, proposed by the national medico-military Department of Special Emergencies.

The State of Emergency has been enforced in Romania in 2020, allowing doctors to apply simplified specific criteria, in order to shorten their physical contact with the patients and to limit staff exposure. Different scores of severity have been used, which determined three-degree severity scale: mild (symptoms of acute upper respiratory tract infection), moderate (documented pneumonia without severity factors) and severe/critical (multiple organ failure syndrome, sepsis, $\mathrm{O}_{2}$ saturation $\leq 93 \%$, respiratory rate $\geq 30 \mathrm{rpm}$ ), provided by the Decree no. 860/2020 (Ministry of Health, 2020). Although numerous studies have demonstrated the relevance of such scores on a general population scale, their lack of sensitivity or specificity has been repeatedly reported (Robert et al., 2020). Our study showed that $46 \%$ of cases developed lung complications, such as COVID-19 pneumonia, which led to a longer hospitalization (an average of 9.62 days).

Another difficult ethical aspect could be the fact that hospitalized patients could not be visited by family, a reluctantly accepted decision, pending a good therapeutic response, followed by discharge, in most cases.

Last but not least, SARS-CoV-2 pandemic had a major impact on medical staff in terms of pressure and overuse. Work overload, unequal distribution of resources and emotional exhaustion may lead to distress and burnout in high risk populations, such as healthcare professionals directly involved in managing patients with SARS-CoV-2 infection (Ilias et al., 2021).

\section{Conclusions}

Management of patients with SARS-CoV-2 infection in the dynamics of the current epidemiological context is difficult and represents a real challenge. Therefore, a good interdisciplinary collaboration is necessary especially in these difficult times.

Further clinical and research studies are necessary to maximize therapeutic effectiveness of current recommended medication, according to national and international guidelines, within an ethical framework. 


\section{References}

Blot, F., Dumont, S. N., Vigouret-Viant, L., Verotte, N., Rossignol, J., Rieutord, A. \& Altea, A. (2020). Ethical issues related to the COVID-19 pandemic in patients with cancer: experience and organisations in a French comprehensive cancer centre. BMJ supportive \& palliative care.

Cucinotta, D. \& Vanelli, M. (2020). WHO Declares COVID-19 a Pandemic. Acta Biomed, 91(1), 157-160.

Ilias Y., Mantziou V., Vamvakas E., Kampisiouli E., Theodorakopoulou M., Vrettour C., ... Dimopoulou I. (2021). Post-traumatic stress disorder and burnout in healthcare professionals during the SARS-CoV-2 pandemic: a cross-sectional study. The Journal of Critical Care Medicine, 7(1), 14-20.

Lai, C. C., Shih, T. P., Ko, W. C., Tang H. J. \& Hsueh, P. R. (2020). Severe acute respiratory syndrome coronavirus 2 (SARS-CoV-2) and coronavirus disease-2019 (COVID-19): The epidemic and the challenges. Int J Antimicrob Agents, 55(3), 1059.

Laventhal, N., Basak, R., Dell, M. L., Diekema, D., Elster, N., Geis, G. \& Macauley, R. (2020). The Ethics of Creating a Resource Allocation Strategy during the COVID-19 Pandemic. Pediatrics, 146(1).

Ministry of Health. (2020). Decree no. 860/2020 regarding modification of Ministry of Health Decree no. 487/2020 for approving the therapeutic protocol of SARS-CoV-2 infection. Official Monitor of Romania no. 432/22.05.2020.

Ministry of Health. (2020). Decree no. 1137/2020 regarding modification of Ministry of Health Decree no 555/2020 for approving the Plan of Measures in order to preparate the hospitals for the COVID-19 pandemia. Official Monitor of Romania no. 539/23.06.2020.

Moderbacher, C. R., Ramirez, S. I., Dan, J. M., Grifoni, A., Hastie M. K., Weiskopf, D. \& Crotty S. (2020). Antigen-Specific Adaptive Immunity to SARS-CoV-2 in Acute COVID-19 and Associations with Age and Disease Severity. Cell, 183(4), 996-1012.

President of Romania. (2020). Decree no. 240/2020 regarding the prolongation of Emergency State in Romania. Official Monitor of Romania no. 311/14.04.2020.

Robert, R., Kentish-Barnes, N., Boyer, A., Laurent, A., Azoulay, E. \& Reignier, J. (2020). Ethical dilemmas due to the Covid-19 pandemic. Ann Intensive Care, 10, 84.

Romanian Government, Communication Group of National Coordination Committee for COVID-19 Vaccination Activities. (2021). Press release, Bucharest, 15.01.2021. https://vaccinare-covid.gov.ro/wp-content/uploads/2021/01/COMUNICAT-pers. vacc.-Et.-115.01.21.pdf

Turale, S., Meechamnan, C., Kunaviktikul, W. (2020). Challenging times: ethics, nursing and the COVID-19 pandemic. Int Nurs Rev, 67(2), 164-167.

Yepes-Temiño, M. J., Callejas González, R., \& Álvarez Avello, J. M. (2021). A moment for reflection. Ethical aspects in the pandemic SARS-CoV-2/COVID-19 in our clinical practice. Revista espanola de anestesiologia y reanimacion, 68(1), 28-36. 